\title{
LOCALLY SYMMETRIC DESIGNS
}

A design (or balanced incomplete block design) $D$ is locally symmetric if, for any point $p$, the lines and blocks through $p$ form a symmetric design $D^{*}(p)$. Equivalently, $D$ is locally symmetric if the number of blocks containing three non-collinear points and the number of points in two nondisjoint blocks are both nonzero constants. The main result of this paper is:

THEOREM 1. If $D$ is a locally symmetric design with parameters $v, k$, in which a line has $s+1$ points and three non-collinear points are contained in $t$ blocks, then exactly one of the following occurs:

(i) $D$ is the design of points and hyperplanes in a projective geometry of dimension at least 3 over $G F(s)$;

(ii) $D$ is the design of points and hyperplanes in an affine geometry of dimension at least 3 over $G F(s+1), s>1$;

(iii) $D$ is a Hadamard 3-design $(s=1, v=4(t+1), k=2(t+1), \lambda=2 t+1$;

(iv) $v=(1+s t)\left(2 s^{2}+2 s+1+(3 s+2) s^{2} t+s^{4} t^{2}\right), k=(1+s t)\left(1+s+s^{2} t\right)$, $\lambda=1+(2 s+1) t+s^{2} t^{2}$, and if $s>1$ and $t>1$ then $t>s+2$ and $s+1$ divides $t(t-1)$

(v) $t=1, v=(s+1)^{4}\left(s^{3}+2 s^{2}+3 s+1\right), k=(s+1)^{2}\left(s^{2}+s+1\right), \lambda=s^{3}+$ $+3 s^{2}+4 s+3$;

(vi) $s=1, t=3, v=496, k=40, \lambda=39$.

Some special cases of this result have already been proved. It is known, for example, when $D$ is symmetric (Dembowski and Wagner [10]), affine (Dembowski [7], [8]), an extension of a symmetric design (the case $s=1$ : Cameron [4]), locally a projective plane (the case $t=1$ : Doyen and Hubaut [11]), or locally a projective geometry of dimension at least 3 (essentially [11]; this also follows from results of Kantor [16]). Only one example of (iv) is known, the Steiner system $S(3,6,22)$ with $s=t=1$ (Witt [20], [21]); no example of (v) or (vi) is known. Theorem 1 has some interest from the point of view of axiomatisations of finite projective and affine geometries (see [16]).

Similarly, but more easily, it will be shown that a locally affine design (similarly defined) is an inversive plane. This is a generalisation of a result of Dembowski ([9] p. 76).

\section{Definitions}

A design $D$ with parameters $v, k, \lambda(k<v-1, \lambda>0)$ is a set of $v$ points 
together with a family of $k$-subsets of the point set called blocks, with the property that any two points are contained in exactly $\lambda$ blocks. If $b$ is the total number of blocks, and $r$ the number containing any point (this number is constant), then

$$
\begin{aligned}
& b k=v r, \\
& r(k-1)=\lambda(v-1) .
\end{aligned}
$$

Fisher's inequality states that $b \geqslant v$ (equivalently $r \geqslant k$ ). $D$ is symmetric if any one of the equivalent conditions holds:

(i) $v=b$;

(ii) $k=r$;

(iii) any two blocks contain $\lambda$ common points;

(iv) any two blocks contain equally common points.

Thus, in a symmetric design, $k(k-1)=\lambda(v-1)$. The design of points and hyperplanes in a finite projective geometry is symmetric.

A design is called affine if it satisfies the conditions:

(i) two blocks which are disjoint from the same block are disjoint from one another;

(ii) the number of points in two non-disjoint blocks is a constant $\mu$. The design of points and hyperplanes in a finite affine geometry is affine. For proofs and elaborations on these ideas, the reader is referred to Dembowski [9], Chapter 2.

In a design $D$, the line through two points is the intersection of all blocks containing the two points. Two points lie on a unique line. (For if $q$ and $q^{\prime}$ are points on the line $p p^{\prime}$, every block which contains $p$ and $p^{\prime}$ contains $q$ and $q^{\prime}$; thus the set of $\lambda$ blocks containing $q$ and $q^{\prime}$ is identical with the set of blocks containing $p$ and $p^{\prime}$, and the lines $p p^{\prime}$ and $q q^{\prime}$ are identical.) An arbitrary intersection of blocks contains the line through any two of its points.

Dembowski ([9], p. 66) calls a design smooth if any three non-collinear points are contained in $t$ blocks, where $t$ is a positive constant. In a smooth design, all lines contain equally many points. For if $L$ is a line and $p, p^{\prime}$ are points of $L$, count in two ways the number of choices of a block containing $p$ and $p^{\prime}$ and a third point in this block:

whence

$$
(|L|-2) \lambda+(v-|L|) t=\lambda(k-2),
$$

$$
|L|=(\lambda k-v t) /(\lambda-t) .
$$

I shall let $s+1$ denote the number of points on a line in a smooth design. A 3-design is a smooth design with $s=1$ (that is, a design in which any three points lie in $t$ blocks). A Hadamard 3-design is a 3-design with parameters 
$v=4(t+1), k=2(t+1),=2 t+1, r=4 t+3, b=8 t+6$. Such a design can be constructed from a Hadamard matrix with a distinguished row or column ([9] pp. 111-113), and is an affine design with $\lambda=t+1$. Conversely, any affine 3-design is a Hadamard 3-design. The design of points and hyperplanes in an affine geometry over $G F(2)$ is a Hadamard 3-design; there are many others.

If $p$ is a point of the smooth design $D$, the configuration $D^{*}(p)$ of lines and blocks through $p$ is a design with parameters $v^{*}=(v-1) / s, k^{*}=(k-1) / s$, $\lambda^{*}=t, r^{*}=\lambda, b^{*}=r$. (We identify a block through $p$ with the set of lines through $p$ it contains.) Thus a smooth design is 'locally a design'. A locally symmetric (or locally affine) design is a smooth design $D$ in which $D^{*}(p)$ is symmetric (or affine) for any point $p$. If $D$ is locally symmetric, then any two blocks of $D^{*}(p)$ have $t$ common 'points' of $D^{*}(p)$, and so two blocks of $D$ which contain $p$ have $1+s t$ common points of $D$. Conversely, if $D$ is smooth and any two blocks of $D$ have 0 or $\mu$ common points, then two blocks of $D^{*}(p)$ have $(\mu-1) / s$ common 'points', and so $D^{*}(p)$ is symmetric, and $\mu=1+s t$. A similar but more elaborate characterisation of locally affine designs could be given. The designs listed under (i)-(iii) of Theorem 1 are locally symmetric.

\section{LOCALLY SYMMETRIC DESIGNS}

Let $D$ be a locally symmetric design, with parameters $v, k, \lambda, r, b, \mu, s, t$ as described in Section 1. In this section I show one of four cases must occur:

(a) $D$ is symmetric;

(b) $D$ is affine;

(c) $v=(1+s t)\left(2 s^{2}+2 s+1+(3 s+2) s^{2} t+s^{4} t^{2}\right), k=(1+s t)\left(1+s+s^{2} t\right)$, $\lambda=1+(2 s+1) t+s^{2} t^{2}$

(d) $v=(1+s)(1+s t)\left(s(s+2) / t+3 s^{3}+6 s^{2}+3 s+1+s^{2}(s+1)(3 s+2) t+\right.$ $\left.+s^{4}(s+1) t^{2}\right), k=(1+s)(1+s t)\left(1+s+s^{2} t\right), \lambda=s+2+(s+1)(2 s+1) t+$ $+s^{2}(s+1) t^{2}$, and $t$ divides $s+2$.

\section{LEMMA 1.}

(i)

(ii) $\lambda(v-1)=r(k-1)$;

(iii) $\quad \lambda(\lambda-1)=t(r-1)$;

(iv) $\quad v=1+s r$;

(v) $\quad k=1+s \lambda$;

(vi) two blocks contain 0 or $\mu=1+$ st points.

Proof. (i) and (ii) are the basic equations connecting the parameters of $D$, (iii) the basic equation for $D^{*}(p)$, and (iv)-(vi) the equivalent conditions 
that $D^{*}(p)$ is symmetric. Note that all parameters can be expressed in terms of $s, t$ and $\lambda$.

Suppose $B$ is any block of $D$. Let $D_{0}(B)$ denote the configuration of points outside $B$ and blocks disjoint from $B$. Clearly $D_{0}(B)$ has $v-k$ points, with $k$ points in any block and 0 or $\mu$ points in any two blocks.

LEMMA 2. $D_{0}(B)$ has $b-1-k(r-1) / \mu$ blocks. Of these, $r-k \lambda / \mu$ contain any point, and the number containing two points $q, q^{\prime}$ is 0 or $(\lambda-t) / \mu$ according as the line $q q^{\prime}$ meets $B$ or not.

Proof. $r-1$ blocks different from $B$ contain each of the $k$ points of $B$; each such block meets $B$ in $\mu$ points, so there are $k(r-1) / \mu$ such blocks, and $b-1-k(r-1) / \mu$ blocks disjoint from $B$. Similarly, through any point $q \notin B$ there are $r-k \lambda / \mu$ blocks disjoint from $B$. Finally, let $q$ and $q^{\prime}$ be two points outside $B$. If the line $q q^{\prime}$ meets $B$, then so does block containing $q$ and $q^{\prime}$. Otherwise each of the $k$ points of $B$ lies in $t$ blocks containing $q$ and $q^{\prime}$, and so the number of blocks of $D_{0}(B)$ containing $q$ and $q^{\prime}$ is

$$
\lambda-k t / \mu=(\lambda(1+s t)-(1+s \lambda) t) /=(\lambda-t) \mu .
$$

A corollary of lemma 2 is that $D$ is symmetric if and only if every line meets every block (since either statement is equivalent to the assertion that any two blocks meet). For the remainder of this section we shall make the assumption that $D$ is not symmetric, and show that one of (b), (c) and (d) must occur.

\section{LEMMA 3. $\mu$ divides $\lambda-t$.}

Proof. There is a line $L$ disjoint from a block $B$; the number of blocks containing $L$ and disjoint from $B$ is $(\lambda-t) / \mu$.

Putting $\lambda-t=u \mu$, we have

$$
\begin{aligned}
v= & (1+s t)\left(t(1+s u)^{2}+u(u-1) s\right) / t \\
b= & \left(t(1+s u)^{2}+u(u-1) s\right)\left(u^{2}-u+2 u t+2 u^{2} s t-u s t+\right. \\
& \left.+t^{2}(1+s u)^{2}\right) / t^{2}(1+s u) \\
k= & (1+s t)(1+s u) \\
r= & \left(u^{2}-u+2 u t+2 u^{2} s t-u s t+t^{2}(1+s u)^{2}\right) / t \\
\lambda= & u+t+u s t \\
\mu= & 1+s t .
\end{aligned}
$$

The parameter sets listed as (c) and (d) are obtained when $u=\mu=1+s t$ and $u=1+(1+s) \mu=2+s+s t+s^{2} t$ respectively. (In (d), the condition $t \mid s+2$ comes from observing that $b$ and $r$ are integers.)

LEMMA 4. If $u>\mu$ then $u=1+(1+s) \mu$. 
Proof. From the expression for $b$, we find that $1+s u$ divides $(1+s)(u-\mu)$. If $u>\mu$, then $(1+s)(u-\mu) \geqslant 1+s u$. If equality holds, then $u=1+(1+s) \mu$. Otherwise,

$$
\begin{aligned}
& (1+s)(u-\mu) \geqslant 2+2 s u, \\
& (s-1) u+(s+1) \mu+2 \leqslant 0,
\end{aligned}
$$

which is impossible.

LEMMA 5. The number of blocks of $D_{0}(B)$ is greater than, equal to, or less than the number of points according as $u$ is greater than, equal to, or less than $\mu$.

Proof. By lemma 2, $D_{0}(B)$ is a 'tactical configuration' with $v_{0}=v-k$ points, $b_{0}=b-1-k(r-1) / \mu$ blocks, $k_{0}=k$ points in a block, and $r_{0}=r-$ $-k \lambda / \mu$ blocks through a point. Since $b_{0} k_{0}=v_{0} r_{0}, b_{0}-v_{0}$ has the same sign as $r_{0}-k_{0}=\lambda(u-\mu) / t$.

\section{LEMMA 6. If $u<\mu$, then $u=1$.}

Proof. Assume $u<\mu$; then $D_{0}(B)$ has more points than blocks. Let $M$ be an incidence matrix of $D_{0}(B)$. ( $M$ is a matrix with rows and columns indexed by points and blocks of $D_{0}(B)$, in some order, with $(q, C)$ entry 1 if $q \in C, 0$ otherwise.) $M M^{\tau}$ has $\left(q, q^{\prime}\right)$ entry equal to the number of blocks of $D_{0}(B)$ containing $q$ and $q^{\prime}$; this number is $r-k \lambda / \mu=u+u(u-1) s+$ $+u(u-1) / t$ if $q=q^{\prime}$, and is 0 or $(\lambda-t) / \mu=u$ if $q=q^{\prime}$. So

$$
M M^{\mathfrak{r}}=(u+u(u-1) s+u(u-1) / t) I+u P,
$$

where $P$ is a symmetric matrix with entries 0 and 1 .

$M$ has more rows than columns, so $M M^{\tau}$ is singular. Let $\pi$ be the eigenvalue of $P$ corresponding to the eigenvalue 0 of $M M^{\tau} . \pi$ is an algebraic integer, and

$$
\pi=-1-(u-1) s-(u-1) / t
$$

is rational, so $\pi$ is a rational integer; that is, $t$ divides $u-1$. Put $u=1+x t$ for some integer $x$; since $u<\mu, x<s$.

From the formula for $b, t^{2}(1+s u)$ divides

$$
\begin{aligned}
& \left(t(1+s u)^{2}+u(u-1) s\right)(u(u-1)+2 u t(1+s u)-u s t- \\
& \left.+t^{2}(1+s u)^{2}\right) \\
& =t^{2}\left((1+s u)^{2}+u x s\right)\left(u x+2 u(1+s u)-u s-t(1+s u)^{2}\right),
\end{aligned}
$$

and so $1+s u$ divides $u x s(u x-u s)$. Since $1+u s$ and $u^{2} s$ are coprime, $1+s u=$ $=1+s+s x t$ divides $x(s-x)$. Since $1+s+s x t>x(s-x) \geqslant 0$, we must have $x(s-x)=0, x=0, u=1$.

LEMMA 7. If $u=1$, then $D$ is affine. 
Proof. Assume $u=1$. By lemma 2, a point not incident with a block $B$ is incident with a unique block disjoint from $B$; so two blocks disjoint from $B$ are disjoint. Also, two blocks which are not disjoint have $\mu$ common points. So $D$ is affine.

\section{LINEAR SPACES}

A linear space consists of a set of points together with a collection of subsets called lines, each containing at least two points, with the property that any two points lie in a unique line. A linear subspace (or linear variety) is a subset of the point set which contains the line joining any two of its points. (If every line has just two points, then any set of points is a linear subspace.) A projective plane is a linear space with $s+1$ points on a line $(s>1)$ in which any two lines intersect (equivalently, a linear space which is also a symmetric design, or a linear space with $s+1$ points on a line and $s^{2}+s+1$ points altogether). An affine plane is a linear space which is also an affine design (equivalently, a linear space with $s+1$ points on any line $(s+1)^{2}$ points altogether). In any linear space, a linear plane is a minimal linear subspace containing three non-collinear points. Any projective plane, or any affine plane with more than two points on a line, is a linear plane.

The projective geometry of dimension $d$ over a skew field $F$ is the collection of subspaces of a vector space of rank $d+1$ over $F$. An $i$-dimensional subspace of the geometry is a vector subspace of rank $i+1$; points, lines, planes, and hyperplanes are subspaces of dimension $0,1,2, d-1$ respectively. The points and lines form a linear space, in which the linear subspaces are just the (geometrical) subspaces. If $F$ is finite and $1 \leqslant i \leqslant d-1$, the points and $i$-dimensional subspaces form a design, in which the lines are precisely the (geometrical) lines. Veblen and Young ([18], volume 1) showed that a linear space is a projective geometry of dimension at least 3 if and only if it is not a plane but all its linear planes are projective planes.

If $D$ is a linear space with $s+1$ points on any line, then a linear plane of $D$ has at least $s^{2}+s+1$ points. (For given a point $p$ and a line $L$, with $p \notin L$, the linear plane containing $p$ and $L$ contains every line joining $p$ to a point of $L$.) If $s>1$ and every linear plane has $s^{2}+s+1$ points, then every linear plane is a projective plane, and so $D$ is a projective geometry (if it is not a plane).

Suppose $D$ is a smooth design with $s+1$ points on each line, and $p$ is a point of $D$. Let $L^{*}$ be a line of $D^{*}(p)$. $L^{*}$ is a collection of lines of $D$; let $P\left(L^{*}\right)$ be their union. Then $\left|P\left(L^{*}\right)\right|=1+s\left|L^{*}\right|$. Furthermore, $P\left(L^{*}\right)$ is the intersection of all blocks containting three non-collinear points of $D$, so $P\left(L^{*}\right)$ contains a linear plane of $D$. Thus $\left|P\left(L^{*}\right)\right| \geqslant s^{2}+s+1$, whence $\left|L^{*}\right| \geqslant s+1$. If $s>1$ and every line of each design $D^{*}(p)$ has $s+1$ points, then 
the linear space of $D$ is a projective geometry, and the blocks of $D$ are subspaces of the geometry.

LEMMA 8. If $\mathscr{B}$ is a set of subspaces of a projective geometry forming a symmetric design on the point set, then $\mathscr{B}$ is the set of all hyperplanes.

Proof. Suppose the geometry has dimension $v-1$. Every member of $\mathscr{B}$ has the same dimension, say $k-1$, and every intersection of two members of $\mathscr{B}$ has the same dimension, say $\lambda-1$. If the underlying field is $G F(q)$, then the symmetric design has parameters $V=\left(q^{v}-1\right) /(q-1), K=$ $=\left(q^{k}-1\right) /(q-1), A=\left(q^{\lambda}-1\right) /(q-1)$.

We have

$$
\begin{aligned}
& (V-1) \Lambda=K(K-1), \\
& \left(q^{v-1}-1\right)\left(q^{\lambda}-1\right)=\left(q^{k}-1\right)\left(q^{k-1}-1\right), \\
& q^{v+\lambda-1}-q^{v-1}-q^{\lambda}=q^{2 k-1}-q^{k}-q^{k-1} .
\end{aligned}
$$

The highest power of $q$ dividing the left-hand side is $q^{\lambda}$, whereas the highest power dividing the right-hand side is $q^{k-1}$. So $\lambda=k-1, k=v-1$, and the blocks are hyperplanes. Since there are $\left(q^{v}-1\right) /(q-1)$ hyperplanes, the result is proved.

Remark. The concept of a design can be generalised: if $F$ is a finite field, a design over $F$ can be defined as a collection of blocks (subspaces of rank $k$ ) in a vector space of rank $v$ over $F$, with the property that any subspace of rank 2 is contained in $A$ blocks, where $A>0, k<v-1$. This can be further generalised: we could define a $t$-design over $F$ by analogy with the classical definition [15]. Fisher's inequality and the characterisation of symmetric designs given in Section 1, together with recent generalisations to $t$-designs [19], [5], extend easily to this situation; so we could define a 'symmetric design over $F$ ' in the obvious way. However, Lemma 8 shows that 'symmetric designs over finite fields' do not exist.

LEMMA 9. Suppose $D$ is a locally symmetric design with $s+1$ points on a line $(s>1)$. Suppose any line of any design $D^{*}(p)$ also has $s+1$ points. Then $D$ is the design of points and hyperplanes in a projective geometry of dimension at least 3 over $G F(s)$.

Proof. We have remarked that the linear space derived from $D$ is a projective geometry (over $G F(s)$ ), and the blocks of $D$ are subspaces of this geometry. $D^{*}(p)$ then satisfies the hypotheses of Lemma 8 , and so is the design of points and hyperplanes in a projective geometry of dimension (say) $v-1$ over $G F(s)$; then $r=\left(s^{v}-1\right) /(s-1), \lambda=\left(s^{v-1}-1\right) /(s-1)$, $t=\left(s^{v-2}-1\right) /(s-1)$. Then $1+s t>s^{v-2}=\lambda-t$; so by lemma $3, D$ is symmetric, and itself satisfies the hypotheses of Lemma 8.

The affine geometry of dimension d over a skew field $F$ is obtained from 
the projective geometry by deleting a hyperplane (the 'hyperplane at infinity') together with all its subspaces. Many properties of affine geometries are similar to those of projective geometries, but there are significant differences. The points and lines of an affine geometry form a linear space; if $|F|>2$, the linear subspaces are precisely the geometric subspaces. (This is false when $F=G F(2)$, since then any line has two points.) If a linear space which is not a plane has $s+1$ points on a line $(s>1)$ and any four noncoplanar points lie in a 3-dimensional affine space, then the linear space is an affine geometry; but Hall has given an example of a linear space which is not an affine geometry in which any three non-collinear points lie in an affine plane [12]. His example has three points on any line. On the other hand, Buekenhout [3] has shown that a linear space with at least four points on a line, in which every linear plane is an affine plane, is an affine geometry. Furthermore, Hall [13] showed that a Steiner triple system (a linear space with three points on a line) in which every linear plane is an affine plane, can be described by a commutative Moufang loop of exponent 3. Bruck ([2], p. 157) has shown that such a loop is centrally nilpotent; it follows that the number of points is a power of 3 .

\section{COMPLETION OF THE PROOF AND SOME REMARKS}

Suppose $D$ is a locally symmetric design, as in Section 2 . We have seen that one of the cases (a)-(d) of that section occurs. From [7], [8], and [10] it follows that, if $D$ is symmetric, then conclusion (i) of Theorem 1 holds, and if $D$ is affine then (ii) or (iii) holds. (These results can be proved using the machinery we have set up, the first very easily.) So we shall now assume we are in case (c) or (d).

A non-empty intersection of two blocks is a linear subspace with $1+s t$ points, and is not a line unless $t=1$; so if $t>1$ then $t \geqslant s+1$. Furthermore, if $s>1$ then $t \neq s+1$ by Lemma 9, and so $t \geqslant s+2$. Also, the number of lines in this linear space is $(1+s t) s t /(1+s) s$, so $s+1$ divides $t(1+s t)$, whence $s+1$ divides $t(t-1)$.

Suppose $t=s+2$ and $s>1$ in (c) or (d). The intersection of two blocks has $(1+s)^{2}$ points and so is an affine plane. Since $s>1$, all the linear planes are affine planes. If $s>2$ we have seen that the linear space is an affine geometry, and so it has $(s+1)^{d}$ points, for some integer $d$; we have seen that the second conclusion also holds if $s=2$. But substitution shows that, if $t=s+2$,

$$
\begin{aligned}
& v=(s+1)^{2}\left(s^{6}+4 s^{5}+7 s^{4}+8 s^{3}+6 s^{2}+2 s+1\right) \text { in case (c), } \\
& v=(s+1)^{4}\left(s^{6}+4 s^{5}+7 s^{4}+8 s^{3}+7 s^{2}+3 s+1\right) \text { in case (d). }
\end{aligned}
$$

So these cases cannot arise. We must have $s=1$ or $t=1$ or $t>s+2$. 
If case (d) occurs then $t$ divides $s+2$. The case $t=1$ yields ( $v$ ) of Theorem 1, $s=1$ and $t>1$ yields (vi), and $t>s+2$ is obviously impossible. In case (c) we obtain (iv).

Remarks. (1) Let $D$ be locally symmetric but not symmetric, $B$ a block, and $q$ a point outside $B$. The block $B$ can be 'projected' into $D^{*}(q)$; its image (the set of lines joining $q$ to points of $B$ ) is a set of points of $D^{*}(q)$ with the property that any block of $D^{*}(q)$ meets it in 0 or $\mu$ points. (In particular, if $t=1$, then the image of $B$ is a $\{\mu(1+s u), \mu\}$-arc in the projective plane $D^{*}(q)$ of order $u \mu$, in the sense of Barlotti [1]). It is worth noting also that the set of blocks containing $q$ but disjoint from $B$ becomes, in the dual of $D^{*}(q)$, a set of points which meets any block in 0 or $u$ points. Furthermore, the projections of all blocks not containing $q$ form the blocks of a design $D^{\prime}(q)$ with parameters $v^{\prime}=(v-1) / s, k^{\prime}=k, \lambda^{\prime}=s^{2}(\lambda-t), r^{\prime}=s(r-\lambda), b^{\prime}=b-r$, on the points of $D^{*}(q)$.

(2) The Bruck-Ryser-Chowla theorem ([9], pp. 61, 63), applied to $D^{*}(p)$, eliminates some values of $s$ in (v), but no possibilities in any other case. The only remaining case of (iv)-(vi) to be settled is (iv) with $s=t=1$, where Witt [20], [21] showed the existence of a unique example. Other small cases have received attention, usually by applying the last remark. As observed in [11], Cossu [6] showed that the Desarguesian plane of order 9 contains no $\{21,3\}$-arc; so in (iv) with $s=2, r=1$, any $D^{*}(p)$ must be a non-Desarguesian plane of order 9. (This was also proved by Assmus (personal communication).) A symmetric design with the appropriate parameters for $D^{*}(p)$ in (iv) with $s=1, t=2$ (namely $v^{*}=56, k^{*}=11, \lambda^{*}=2$ ) is constructed in [14]; but, by considering sets of 12 points in this design meeting every block in 0 or 3 points, Baumert and Hall showed it could not occur in this situation (Hall, personal communication). In (v) with $s=1, D^{*}(p)$ is a projective plane of order 10 , and a block not containing $q$ projects to a $\{12,2\}$-arc, or hyperoval; such sets have been important in recent investigations into the existence of a plane of order 10 [17]. In case (iii), however, it is conjectured that a Hadamard 3-design exists with any given value of $t$. Many examples are known.

(3) For a given value, greater than 1 , of $t$, there are only finitely many sets of parameters. This generalises as strongly as possible a result of Hughes [15], who proved it for $s=1$ without the restriction $t>1$. However, there are infinitely many designs which are 'locally projective planes', for example, the projective and affine geometries of dimension 3 ; so the condition $t>1$ is necessary in general.

\section{LOCALLY AFFINE DESIGNS}

An affine design $D^{*}$ has parameters $v^{*}=\mu m^{2}, k^{*}=\mu m, \lambda^{*}=(\mu m-1) /(m-1)$, 
$r^{*}=\left(\mu m^{2}-1\right) /(m-1), b^{*}=m\left(\mu m^{2}-1\right) /(m-1)$, where $m-1$ divides $\mu-1$; here $\mu$ is the number of points in two non-disjoint blocks, and $m$ the number of blocks in a 'parallel class'. (See [9] p. 73.) An affine plane is an affine design with $\lambda^{*}=1$ (equivalently $\mu=1$ ). An inversive plane (or Möbius plane) is an extension of an affine plane, that is, a 3-design which is locally an affine plane ([9] chapter 6). An inversive plane is a 3-design with parameters $v=m^{2}+1$, $k=m+1, t=1$, and conversely.

THEOREM 2. A locally affine design is an inversive plane.

Proof. Suppose $D$ is locally affine and $D^{*}(p)$ has the parameters given above. If $D$ has parameters $v, k, \lambda, r, b$, any three non-collinear points lie in $t$ blocks, and a line has $s+1$ points, then

$$
\begin{aligned}
t & =(\mu m-1) /(m-1) \\
\lambda & =\left(\mu m^{2}-1\right) /(m-1) \\
r & =m\left(\mu m^{2}-1\right) /(m-1) \\
v & =1+s \mu m^{2} \\
k & =1+s \mu m
\end{aligned}
$$

and any two blocks have 0,1 , or $1+s \mu$ common points. Then

$$
b=v r / k=\left(1+s \mu m^{2}\right) m\left(\mu m^{2}-1\right) /(m-1)(1+s \mu m) ;
$$

thus $1+s \mu m$ divides $\left(1+s \mu m^{2}\right) m\left(\mu m^{2}-1\right)$, and so $1+s \mu m$ divides $(m-1)$ $(m+s)$. Suppose $\mu \neq 1$. Since $m-1$ divides $\mu-1, \mu \geqslant m$, and

$$
\begin{aligned}
& 1+s m^{2} \leqslant m^{2}+s m-m-s, \\
& (s-1)\left(m^{2}-m+1\right) \leqslant-2, \text { which is impossible. }
\end{aligned}
$$

Thus $\mu=1$ and $D$ is locally an affine plane.

By Fisher's inequality, $r \geqslant k$, or $m(m+1) \geqslant 1+s m$, whence $s \leqslant m$. Also $1+s m$ divides $m^{2}+s m-m-s$. Multiplying by $s$ and putting $m s \equiv-1$, $1+s m$ divides $-m-s+1-s^{2}$. If $s^{2}+s+m-1 \leqslant 2+2 s m$, then

$$
\begin{aligned}
& s(2 m-s-1) \leqslant m-3, \\
& 2(m-1) \leqslant m-3,
\end{aligned}
$$

which is absurd. So

$$
\begin{aligned}
s^{2}+s+m-1=1 & +s m, \\
(s-1)(s-m+2) & =0 \\
s & =1 \text { or } m-2
\end{aligned}
$$


If $m=s+2$ then $b=\left(s^{2}+3+1\right)(s+2)(s+3) /(s+1)$, so $s+1$ divides 2 . Thus $s=1$ in any case, and $D$ is an inversive plane.

\section{ACKNOWLEDGEMENT}

The incentive for this work was provided by a weekly seminar at the University of Michigan in the winter term 1973. I am grateful to the University for this opportunity, as well as to Merton College, Oxford (where much of the preliminary work was done) for leave of absence. I am also grateful to W. M. Kantor for showing me an improvement to the main theorem, and to E. F. Assmus Jr. for discussions on the case $t=1$, while I was travelling in the USA.

\section{BIBLIOGRAPHY}

[1] Barlotti, A.: 'Sui $\{k, n\}$ archi di un piano lineare finito', Boll. Un. Mat. Ital. 11 (1956), 553-556.

[2] Bruck, R. H.: A Survey of Binary Systems, Springer-Verlag, Berlin-GöttingenHeidelberg, 1958.

[3] Buekenhout, F.: 'Une caractérisation des espaces affines basée sur la notion de droite', Math. $Z .111$ (1969), 367-371.

[4] Cameron, P. J.: Extending Symmetric Designs, J. Combinational Theory A14 (1973), 215-220.

[5] Cameron, P. J.: 'Near-Regularity Conditions for Designs', Geometriae Dedicata, 2 (1973), 213-223.

[6] Cossu, A.: 'Su alcune proprietà dei $\{k, n\}$ archi di un piano proiettivo sopra un corpo finito', Rend. Mat. e Appl. 20 (1961), 271-277.

[7] Dembowski, P.: 'Eine Kennzeichnung der endlichen affinen Räume', Archiv Math. 15 (1964), 146-154.

[8] Dembowski, P.: 'Berichtigung und Ergänzung zu "Eine Kennzeichnung der endlichen affinen Räume"', Archiv Math. 18 (1967), 111-112.

[9] Dembowski, P.: Finite Geometries, Springer-Verlag, Berlin-Heidelberg-New York, 1968.

[10] Dembowski, P. and Wagner, A.: 'Some Characterisations of Finite Projective Spaces', Archiv Math. 11 (1960), 465-469.

[11] Doyen, J. and Hubaut, X.: 'Finite Regular Locally Projective Spaces', Math. $Z$. 119 (1971), 83-88.

[12] Hall, M., Jr.: 'Automorphisms of Steiner Triple Systems', IBM J. Res. Develop. $4(1960), 460-471$.

[13] Hall, M., Jr.: 'Group Theory and Block Designs', Proc. Int. Conf. Theory of Groups (ed. by L. G. Kovacs and B. H. Neumann), 115-144. Gordon and Breach, New YorkLondon, 1967.

[14] Hall, M., Jr., Lane, R., and Wales, D.: 'Designs Derived from Permutation Groups', J. Combinational Theory 8 (1970), 12-22.

[15] Hughes, D. R.: 'On t-Designs and Groups', Am. J. Math. 87 (1965), 761-778.

[16] Kantor, W. M.: 'Characterisations of Finite Projective and Affine Spaces', Canad. J. Math. 21 (1969), 64-75.

[17] McWilliams, F. J., Sloane, N. J. A., and Thompson, J. G.: 'On the Existence of a Projective Plane of Order 10', J. Combinatorial Theory A14 (1973), 66-78.

[18] Veblen, O. and Young, J. W.: Projective Geometry, Ginn \& Co., Boston, 1916.

[19] Wilson, R. M. and Ray-Chaudhuri, D. K.: 'Generalisation of Fisher's Inequality to t-Designs', Am. Math. Soc. Notices 18 (1971), 805. 
[20] Witt, E.: 'Die 5-fach transitiven Gruppen von Mathieu', Abh. Math. Seminar Hamburg 12 (1938), 256-264.

[21] Witt, E.: 'Uber Steinersche Systeme', Abh. Math. Seminar Hamburg 12 (1938), 265-275.

Author's address:

Dept of Mathematics, University of Michigan, Ann Arbor, Mich. 48104, U.S.A.

Merton College, Oxford OX1 4JD, England

(Received May 2, 1973; in revised form June 22, 1973 